\title{
Loop Amplitudes Induced by Tensor Fermionic Current in Constant Homogeneous Electromagnetic Fields
}

Alexanda A. Dobrynina, ${ }^{a}$ Ilya V. Karabanov, ${ }^{a}$ Alexander Ya. Parkhomenko ${ }^{a, *}$ and Lubov A. Vassilevskaya ${ }^{b}$

${ }^{a}$ P. G. Demidov Yaroslavl State University, Sovietskaya 14, 150003 Yaroslavl, Russia

${ }^{b}$ Fulda University of Applied Sciences, D-36037 Fulda, Germany

E-mail: dobrynina@uniyar.ac.ru, karabanovilya@rambler.ru, parkh@uniyar.ac.ru

The non-diagonal correlator of vector and tensor fermionic currents is considered as the concrete example of the two-point one-loop amplitudes modified by a constant homogeneous magnetic field. The crossed-field limit of this correlator is found. The tensor current is a fermionic part of the Pauli Lagrangian relevant for the electromagnetic interaction of fermions through the anomalous magnetic moment. Under assumption that this interaction enters the effective QED Lagrangian, the contribution to the photon polarization operator linear in AMM is calculated.

40th International Conference on High Energy physics - ICHEP2020

July 28 - August 6, 2020

Prague, Czech Republic (virtual meeting)

${ }^{*}$ Speaker 
The presence of an extreme active medium, for example in astrophysical objects or in heavyion collisions, can influence quantum processes inside or properties of participating particles. Changes in dispersion relations, mass shift for massive particles, appearance of an effective mass for massless particles, or modification of the anomalous magnetic moment are good examples of such an influence induced by effects of a vacuum modified by an external electromagnetic field.

Let us assume that the Lagrangian density of local generalized fermion interaction has the form [1]:

$$
\mathcal{L}_{\text {int }}(x)=\left[\bar{f}(x) \Gamma^{A} f(x)\right] J_{A}(x)
$$

where $J_{A}$ is the generalized current like a photon, neutrino current, etc. Interaction constants are included into the current $J_{A} . \Gamma_{A}$ in (1) is any of $\gamma$-matrices from the set $\left\{1, \gamma_{5}, \gamma_{\mu}, \gamma_{\mu} \gamma_{5}, \sigma_{\mu \nu}=\right.$ $\left.i\left[\gamma_{\mu}, \gamma_{\nu}\right] / 2\right\}$. Two-point correlation function of general form is as follows [1]:

$$
\Pi_{A B}=\int d^{4} X \mathrm{e}^{-i(q X)} \operatorname{Sp}\left\{S_{\mathrm{F}}(-X) \Gamma_{A} S_{\mathrm{F}}(X) \Gamma_{B}\right\}
$$

where $X^{\mu}=x^{\mu}-y^{\mu}$ is the integration variable, $q$ is the momentum entering the correlator, $S_{\mathrm{F}}(X)$ is the Lorentz-invariant part of exact fermion propagator in an external field. Correlations of scalar, pseudoscalar, vector and axial-vector fermionic currents were studied in [2]. We extend this analysis and consider correlations of a tensor current with the other ones. Some preliminary results were published in [3-5].

In QED, the photon polarization operator $\mathcal{P}^{\mu \nu}(q)$ is related with the $\gamma \rightarrow \gamma$ transition matrix element and, consequently, with the correlator of two vector fermionic currents:

$$
\mathcal{M}_{\gamma \rightarrow \gamma}=-i \varepsilon_{\mu}^{\prime *}(q) \mathcal{P}^{\mu \nu}(q) \varepsilon_{\nu}
$$

Dispersion relations for the photon follow from the equations $(\lambda=1,2,3)$ :

$$
q^{2}-\Pi^{(\lambda)}(q)=0,
$$

where $\Pi^{(\lambda)}(q)$ are eigenvalues of the field-induced part of the photon polarization operator: (here, we restrict ourselves with the electron loop only):

$$
\mathcal{P}_{\mu \nu}(q)=\sum_{\lambda=1}^{3} \frac{b_{\mu}^{(\lambda)} b_{\nu}^{(\lambda)}}{\left(b^{(\lambda)}\right)^{2}} \Pi^{(\lambda)}(q) .
$$

Here, $b_{\mu}^{(\lambda)}$ are basic vectors diagonalizing $\mathcal{P}_{\mu \nu}(q)$. As in vacuum, there are two physical eigenstates of $\mathcal{P}_{\mu \nu}(q)$ in an external constant homogeneous magnetic field [1]:

$$
\varepsilon_{\mu}^{(1)}=b_{\mu}^{(1)} / \sqrt{q_{\perp}^{2}}, \quad \varepsilon_{\mu}^{(2)}=b_{\mu}^{(2)} / \sqrt{q_{\|}^{2}} .
$$

As mentioned above, an external electromagnetic field gives additional contribution to the anomalous magnetic moment (AMM) of the fermion. At the Lagrangian language, it can be included as a Pauli term which describes a fermion-photon interaction through the AMM. Within this approach, we calculated a linear term in AMM to the photon polarization operator as a correlator of a vector current with a tensor one. This modifies the field-induced part of $\Pi^{(\lambda)}(q)$ as follows:

$$
\Pi^{(\lambda)}(q)=-i \mathcal{P}\left(q^{2}\right)-\frac{\alpha}{\pi} Y_{V V}^{(\lambda)}+\frac{\alpha}{\pi} a_{e} Y_{V T}^{(\lambda)} .
$$


The last term can be presented in the form of double integral [1]:

$$
Y_{V T}^{(\lambda)}=\int_{0}^{\infty} \frac{d t}{t} \int_{0}^{1} d u\left\{\frac{\beta t}{\sin (\beta t)} y_{V T}^{(\lambda)} e^{-i \Omega}-q^{2} e^{-i \Omega_{0}}\right\}
$$

The resulting integrands appear very simple:

$$
y_{V T}^{(1)}=y_{V T}^{(3)}=q^{2} \cos (\beta t u), \quad y_{V T}^{(2)}=q_{\|}^{2} \cos (\beta t u)-q_{\perp}^{2} \cos (\beta t) .
$$

The part independent on the field is subtracted. For the electron, $a_{e} \sim \alpha=1 / 137$ and the AMM correction is small. This contribution can be enhanced when AMM is dominated by New Physics.

In summary, the correlators of the axial vector fermionic current with the tensor one as well as tensor-tensor correlators in the constant homogeneous magnetic field are also calculated and a corresponding paper is in preparation. Physical applications of these correlators will be also discussed in this paper.

\section{Acknowledgments}

The work of A.D. and I. K. on calculating the vector-tensor correlator in the background magnetic field and its impact into the photon polarization operator is supported by the Russian Science Foundation (Grant No. 18-72-10070). A. P. is partially supported by the YSU Initiative Scientific Research Activity (Project No. AAAA-A16-116070610023-3).

\section{References}

[1] A. Kuznetsov and N. Mikheev, Electroweak processes in external electromagnetic fields, Springer Tracts Mod. Phys. 197 (2004) 1.

[2] M. Borovkov, A. Kuznetsov and N. Mikheev, One loop amplitude of the transition $j \rightarrow f \bar{f} \rightarrow j^{\prime}$ in an external electromagnetic field, Phys. Atom. Nucl. 62 (1999) 1601.

[3] A. Dobrynina, I. Karabanov, A. Parkhomenko and L. Vassilevskaya, Two-Point Correlators of Fermionic Currents in External Magnetic Field, EPJ Web Conf. 158 (2017) 07001.

[4] A. Dobrynina, I. Karabanov, A. Parkhomenko and L. Vassilevskaya, Two-Point One-Loop Fermionic Amplitudes in Constant Homogeneous Magnetic Field, EPJ Web Conf. 201 (2019) 09003.

[5] A. Dobrynina, I. Karabanov, A. Parkhomenko and L. Vassilevskaya, One-loop amplitudes of charged fermions in constant homogeneous electromagnetic fields, J. Phys. Conf. Ser. 1390 (2019) 012075. 